\title{
Erratum
}

\section{Analysis of the Coincidence Method for Comparing Atomic and Nuclear Lifetimes}

\author{
K.W. McVoy, X.T. Tang, and H.A. Weidenmüller \\ Max-Planck-Institut für Kernphysik, Heidelberg, Federal Republic of Germany \\ Z. Phys. A - Atoms and Nuclei 299, 195 (1981)
}

It was kindly pointed out to us by Dr. C. Stoller, ETH Zürich, that we overlooked the following experimental work bearing on the problem:

Chemin, J.F., Andriamonje, S., Roturier, J., Saboya, B., Thibaud, J.P.: International Conference on Atomic Collisions in Solids; Abstracts of Papers; Moscow 1977

Chemin, J.F., Andriamonje, S., Roturier, J., Saboya, B., Thibaud, J.P., Joly, S., Plattard, S., Uzureaù, J., Laurent, H., Maison, J.M., Shapira, J.P.: Nucl. Phys. A 331, 407 (1979)

We apologize to these authors for our oversight, and we thank Dr. Stoller for his communication.

K.W. McVoy

X.T. Tang

H.A. Weidenmüller

Max-Planck-Institut für Kernphysik

Postfach 103980

D-6900 Heidelberg 1

Federal Republic of Germany 